\title{
SOBRE LA PRESENCIA DE TILLANDSIA SCHREITERIY T. SPHAEROCEPHALa (Bromeliaceae) en Argentina
}

\author{
EVA BULACIO ${ }^{1}$ y HUGO AYARDE ${ }^{2}$
}

\begin{abstract}
Summary: On the presence of Tillandsia schreiteri and T. sphaerocephala (Bromeliaceae) in Argentina. Tillandsia sphaerocephala var. tarijensis is mentioned for Argentina for the first time. Diagnostic characters to clearly differentiate it from the typical variety and from $T$. schreiteri, entities considered as synonyms for the argentinean flora, are presented. A detailed description of the new entity, a key, drawings and photographic images are included.
\end{abstract}

Key words: Tillandsia sphaerocephala var. tarijensis, T. schreiteri, Argentinean Flora, Bromeliaceae.

\begin{abstract}
Resumen: En este artículo se menciona por primera vez a Tillandsia sphaerocephala var. tarijensis (Bromeliaceae) para Argentina. Se presentan caracteres diagnósticos para diferenciarla claramente de la variedad típica y de $T$. schreiteri, entidades consideradas, hasta ahora, como sinónimos para la flora argentina. Se incluye una detallada descripción de la novedad, una clave, dibujos e imágenes fotográficas.
\end{abstract}

Palabras clave: Tillandsia sphaerocephala var. tarijensis, T. schreiteri, Flora Argentina, Bromeliaceae.

\section{INTRODUCCIÓN}

Tillandsia schreiteri Lillo \& A. Cast. fue descripta en 1929, sobre material procedente de la provincia de Tucumán (Castellanos, 1929). Posteriormente, esta especie fue referida como sinónimo de $T$. sphaerocephala Baker (Smith, 1970, 1977), una entidad de Bolivia descripta por Baker a finales del siglo XIX y de la que actualmente se reconocen dos variedades: T. sphaerocephala var. sphaerocephala, del centro y norte de Bolivia y T. sphaerocephala var. tarijensis Ehlers \& L. Hrom., del sur de Bolivia. Desde entonces ambas especies son tratadas como sinónimos en la flora de Argentina (Morrone \& Zuloaga, 1996; Schinini et al., 2008; Zuloaga et al., 2013), aún cuando diversos reportes las refieren como entidades diferentes (Ehlers \& Hromadnik, 1998; Krömer et al., 1999), sin advertir que el material procedente de Argentina corresponde exclusivamente a T. schreiteri.

\footnotetext{
1 Laboratorio de Taxonomía Fanerogámica, Fundación Miguel Lillo.4.000 Tucumán. E-mail: evabulacio@yahoo. com.ar

2 Instituto de Ecología, Fundación Miguel Lillo. 4.000 Tucumán.
}

Material procedente de las sierras de Calilegua (Jujuy) y de las Sierras de San Javier (Tucumán) y consultas en el Herbario Nacional de Bolivia (LPB), permitió distinguir inequívocamente ambas especies, a la vez de mencionar por primera vez a T. sphaerocephala var. tarijensis para Argentina, variedad de la que sólo se conocía el tipo.

\section{Resultados}

Tillandsia schreiteri Lillo \& A. Cast. Anales Mus. Nac. Hist. Nat. 36: 56-57. 1929. Fig. 1, A, B у C.

Roseta globosa. Hojas basales numerosas, láminas largamente triangulares de $35-40 \mathrm{~cm}$ long. x 3-5 cm lat. en la base. Eje florífero folioso, con más de 10 hojas, fuertemente inclinado y terminado en una cabezuela con más de 15 espigas.

Distribución y hábitat. Se extiende en áreas montanas húmedas del noroeste de Argentina y sur de Bolivia, entre los 1.100 y $2.300 \mathrm{~m}$ de altitud. En Argentina es común en el bosque montano, entre los 1.100 y los 1.700 m s.n.m. Crece como epifita en la parte superior de árboles de gran porte, 
Bol. Soc. Argent. Bot. 49 (1) 2014
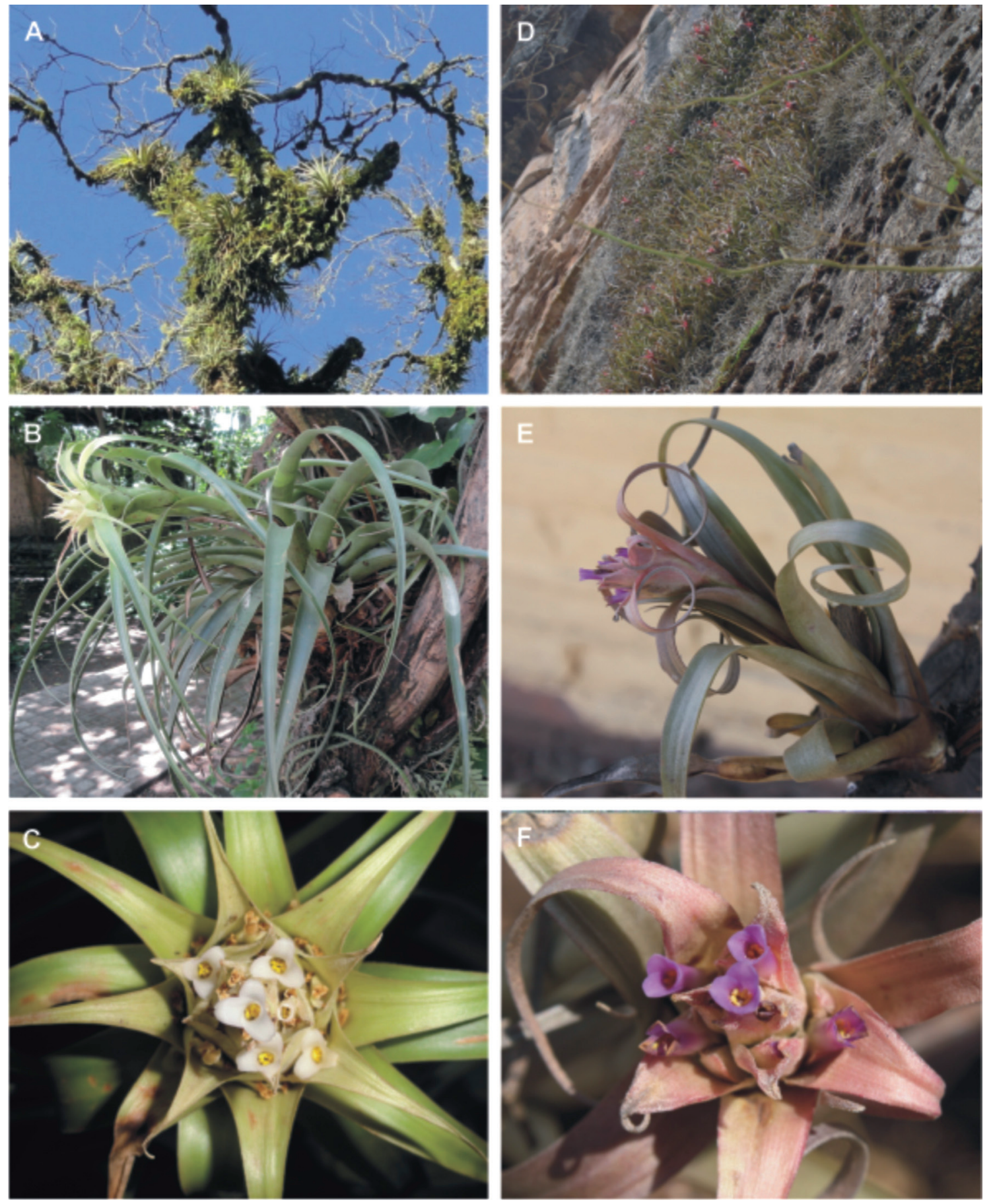

Fig.1. Tillandsia schreiteri $(\mathrm{A}-\mathrm{C})$. A: Planta en su hábitat natural. B: Aspecto de la planta. C: Detalle de la inflorescencia. T. sphaerocephala var. tarijensis (D-F). D: Hábito y hábitat de la variedad. E: Aspecto de la planta. F: Detalle de la inflorescencia. 


\section{E. Bulacio y H. Ayarde - Tillandsia schreiteri y T. sphaerocephala en Argentina}

aunque también se la puede encontrar en las ramas inferiores expuestas y en árboles muertos en pie.

Fenología. Florece entre enero y marzo, en frutos desde abril. El eje florífero, ya seco, puede permanecer en la planta el resto del año.

Obs. Tanto la descripción como la figura presentada en Smith (1977) bajo T. sphaerocephala, en realidad pertenecen a T. schreiteri. Esta confusión probablemente se haya debido a que no observó material fresco ni en campo, esto sumado a la falta de información sobre el color de la flor en la diagnosis de T. sphaerocephala.

Material estudiado. ARGENTINA. Prov. Jujuy: Dpto. Capital, cuesta a Tiraxi, 4-8 km NE de Río Grande, $1.500 \mathrm{~m}, 17 / \mathrm{III} / 1982$, Schinini \& Vanni 22454 (LIL). Prov. Salta: Dpto. Metán, Ea. Gutiérrez, Sa. de Metán, 1.500 m, 29/VIII/78, S. Halloy A-301 (LIL). Prov. Tucumán: Dpto. Lules, Sierras de San Javier: Camino de San Pablo a Villa Nougués, 1.350 m, 02/05/2012, Bulacio \& Ayarde 2490 (LIL); idem, 1.400 m, 14/02/2013, Bulacio \& Ayarde 2715 (LIL). BOLIVIA. Dpto. Santa Cruz, Prov. La Florida: Camino Vallegrande - Mataral, 1.850 m, Ibisch 93-0887 (LPB); Dpto. Tarija, Prov. Arce: Río Cabildo hacia Charaja, $2.350 \mathrm{~m}$, Liberman et al. 1847 (LPB).

Tillandsia sphaerocephala Baker var. tarijensis Ehlers \& L. Hrom. Bromelie 2: 41. 1998. Tipo: BOLIVIA. Dpto. Tarija: Cana Cruz, upper current of Rio Paicho, 2950 m, 6/02/1995, L. Hromadnik 19025 (Holotypus LPB !). Fig. 1, D, E y F; Fig. 2.

Hierba rizomatosa saxícola, de hasta $16 \mathrm{~cm}$ alt. Hojas basales en número de 8 , involutas y dispuestas en roseta; láminas verde grisáceas, blanco lepidotas en ambas superficies, triangulares, largamente atenuadas y terminadas en un ápice revoluto, de 18-25 cm long. x 1,8-3 cm lat. en la base. Inflorescencia terminal que no sobrepasa las hojas, elipsoide, contraída y ligeramente inclinada, de $3 \mathrm{~cm}$ long. x $2 \mathrm{~cm}$ lat., con hasta 6 espigas; pedúnculo cilíndrico, erecto, de hasta 13,5 cm long., cubierto completamente por brácteas foliáceas; las inferiores verde grisáceas, de hasta $20 \mathrm{~cm}$ long.; las superiores, de menor tamaño, rosado verdosas, blanco lepidotas. Brácteas primarias exteriores rosadas, foliáceas, revolutas, anchamente aovadas y largamente acuminadas, mucho más largas que las espigas; brácteas primarias interiores aovadas, ligeramente acuminadas, de igual tamaño o apenas más largas que las espigas. Espigas dísticas, bifloras, oblongas y cortamente pediceladas, de 1,8-3,5 cm long. $x \quad 0,5-0,9 \mathrm{~cm}$ lat.; brácteas florales ampliamente aovadas a elípticas, rosadas con la base verdosa, conspicuamente carinadas y esparcidamente lepidotas. Flores tubulosas, subsésiles; sépalos connados, elípticos agudos en el ápice, de 1,3-1,6 cm long. x 0,5-0,6 cm lat., verdosos en la base, translúcidos, marcadamente carinados y con tricomas glandulosos inmersos en la cara adaxial; pétalos reflexos, libres, violáceos en la porción superior y translúcidos en la inferior, angostamente elípticos con ápice obtuso ligeramente asimétrico, de 1,8-2,2 cm long. x 0,5 $\mathrm{cm}$ lat., con tricomas glandulosos en la cara adaxial; estambres incluidos, filamentos plicados, de 1,2-1,6 cm long.; anteras dorsifijas, amarillas, oblongas; ovario ovoide, verdoso, de $7 \mathrm{~mm}$ long. x $3 \mathrm{~mm}$ de diám.; estilo linear, translúcido, de $8 \mathrm{~mm}$ long.; estigma amarillo. Cápsula parda a negra; semillas fusiformes, pardas con un apéndice plumoso en el ápice.

Distribución y hábitat. Extremo norte de Argentina y sur de Bolivia. En Argentina hasta el presente sólo ha sido encontrada en una única localidad de las sierras de Calilegua, aunque es probable que su distribución sea más extendida. Habita quebradas húmedas del nivel superior del bosque montano, entre los 2.600 y 2.700 m s.n.m. Crece formando colonias sobre paredones de roca caliza.

Fenología. Florece de julio a octubre y los frutos están en dispersión a inicios del verano.

Obs. Se diferencia de la variedad típica, la cual habita en montañas del centro y norte de Bolivia, por el hábito colonial, por el menor porte de la planta y especialmente por el menor número de espigas de la inflorescencia.

Material estudiado. ARGENTINA. Prov. Jujuy: Dpto. Valle Grande, Sierras de Calilegua, Quebrada de Cortaderas, $2650 \mathrm{~m}, 10 / 07 / 2010$, Bulacio \& 
Bol. Soc. Argent. Bot. 49 (1) 2014

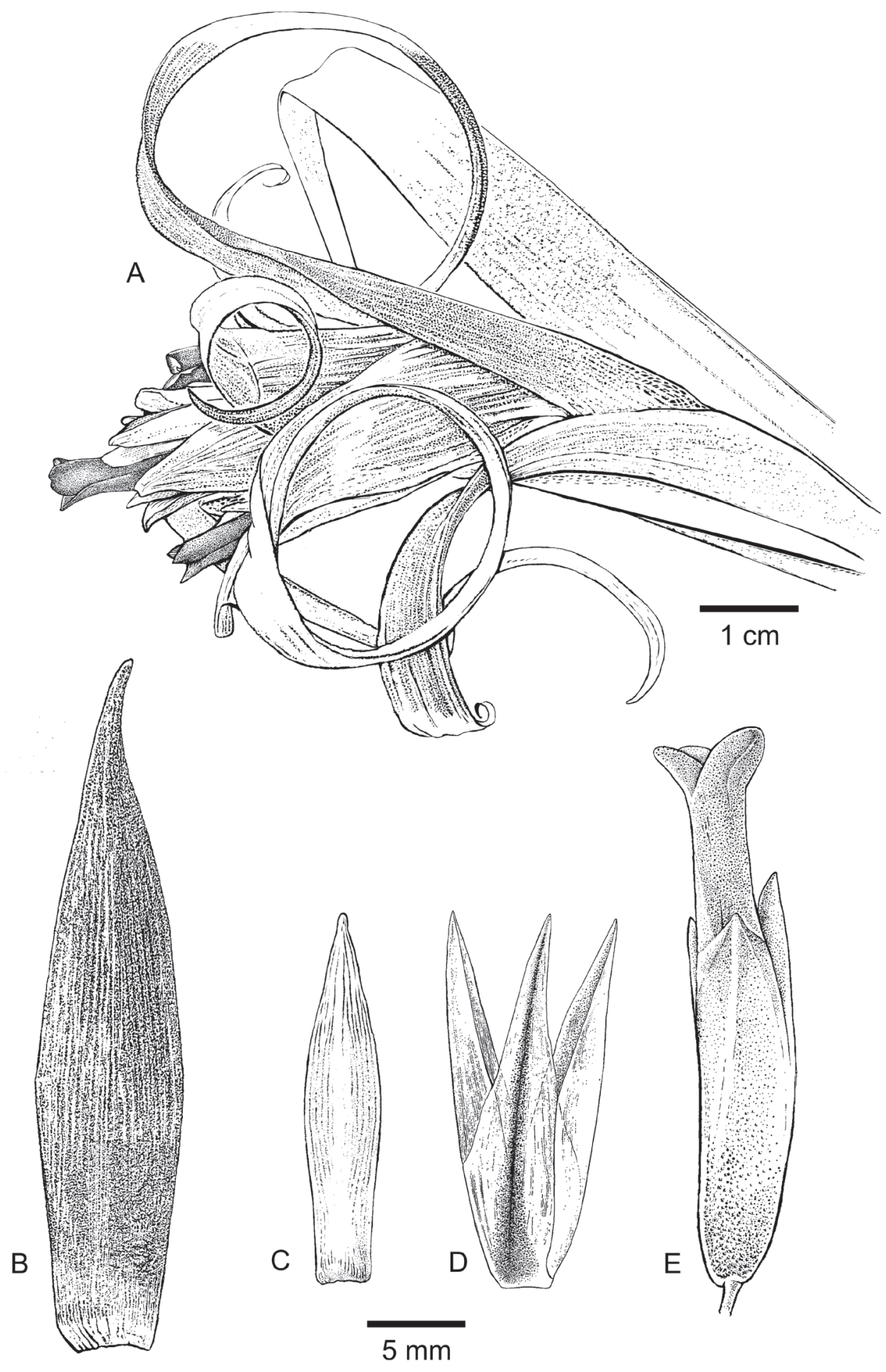

Fig. 2. Tillandsia sphaerocephala var. tarijensis. A: Aspecto de la inflorescencia. B: Bráctea primaria. C: Bráctea floral. D: Detalle del cáliz. E: Detalle de la flor. 


\section{E. Bulacio y H. Ayarde - Tillandsia schreiteri y T. sphaerocephala en Argentina}

Ayarde 1738 (LIL); idem, 08/12/2012, Bulacio \& Ayarde 2495 (LIL).

\section{Clave para diferenciar las entidades}

A. Planta epífita, solitaria o en grupos poco numerosos, de más de $30 \mathrm{~cm}$ de alto, incluyendo la inflorescencia. Hojas basales más de 20, formando una roseta más o menos esférica. Espigas bipinnadas, con más de tres flores por espiga. Pétalos blancos........ Tillandsia schreiteri

A'. Planta saxícola, colonial, de menos de $20 \mathrm{~cm}$ de alto incluyendo la inflorescencia. Hojas basales menos de 10, erectas. Espigas pinnadas, bifloras. Pétalos violáceos.

Tillandsia sphaerocephala var. tarijensis

\section{Agradecimientos}

A la Fundación Miguel Lillo por la provisión de fondos para este estudio, al Dr. Stephan Beck y al personal del Herbario Nacional de Bolivia por la buena disposición durante nuestra visita, a A. Gutiérrez, de la Sección Iconografía de la Fundación Miguel Lillo, por la realización de los dibujos y a los revisores por sus oportunas sugerencias.

\section{Bibliografía}

BAKER, C. F. 1888. A synopsis of Tillandsieae. J. Bot. 26: 14.

CASTELLANOS, A. 1929. Bromeliaceae argentinae novae vel criticae, II. An. Mus. Nac. Hist. Nat. Buenos Aires 36: 49-58.
EHLERS, R. \& L. HROMADNIK. 1998. Eine neue bolivianische Tillandsia aus den Verwandtschaftskreis von. T. sphaerocephala und $T$. schreiteri: T. calochlamys. Bromelie 2: 36-43.

KRÖMER, T., M. KESSLER, B.K. HOLST, H.E. LUTHER, E.J. GOUDA, P.L. IBISCH, W. TILL \& R.VÁSQUEZ. 1999. Checklist of Bolivian Bromeliaceae with notes on species distribution and levels of endemism. Selbyana 20 (2): 201-223.

MORRONE, O \& F.O. ZULOAGA. 1996. Bromeliaceae. In: Zuloaga, F.O. \& O. Morrone (eds.), Catálogo de las plantas vasculares de Argentina, 1. Pteridophyta, Gymnospermae y Angiospermae (Monocotyledoneae), Monogr. Syst. Bot. Missouri Bot. Gard. 60: 106-121.

SCHININI, A., M.G.L. WANDERLEY, T. STREHL, Z. MARTINS \& B. MOREIRA. 2008. Bromeliaceae. In: Zuloaga, F.O., O. Morrone \& M.J. Belgrano (eds.), Catálogo de las plantas vasculares del Cono Sur (Argentina, sur de Brasil, Chile, Paraguay y Uruguay). 1. Pteridophyta, Gymnospermae y Monocotyledoneae. Monogr. Syst. Bot. Missouri Bot. Gard. 107: 245-291.

SMITH, L.B. 1970. Notes on Bromeliaceae XXXI. Phytologia 20: 121-183.

SMITH, L.B. 1977. Bromeliaceae subfamily Tillandsioideae. In: Smith, L. B. \& R. J. Downs, Tillandsioideae. Fl. Neotrop. Monogr. 14(2): 6631492.

ZULOAGA, F. O., O. MORRONE \& M. J. BELGRANO (eds.). Catálogo de las plantas vasculares del Cono Sur. Instituto de Botánica Darwinion. http://.darwin. edu.ar/Proyectos/FloraArgentina/FA.asp. Consulta marzo de 2013.

Recibido el 25 de marzo de 2013, aceptado el 6 de junio de 2013. 
